\title{
CONTRIBUTION A LA RÉALISATION DE SOURCES DU TYPE PENNING POUR LA SPECTROGRAPHIE DANS L'ULTRAVIOLET LOINTAIN
}

\author{
J. L. BOUCHAREL, A. DAUDE, A. QUEMERAIS et S. ROBIN \\ Laboratoire de Spectroscopie, Faculté des Sciences, 35, Rennes
}

(Reçu le 7 novembre 1969)

\begin{abstract}
Résumé. - On décrit quelques sources de type Penning fonctionnant à faible pression (10-4 à $10^{-2}$ torr) et suffisamment stables pour permettre des mesures spectrophotométriques précises dans l'U. V. lointain. Les inconvénients dus à la vaporisation des cathodes ont été réduits. Ces sources sont utilisables notamment pour les mesures en ultravide sans interposition de fenêtre entre la source, le monochromateur et l'enceinte de mesures.
\end{abstract}

Abstract. - Some Penning sources operated at a low pressure (10-4-10-2 torr) and stable enough to permit precise spectrophotometric measurements in the far U. V. are described. The inconvenience caused by the sputtering of the cathodes has been reduced. These sources are particularly suitable for high vacuum measurements made without window between the source, the monochromator and the detector.

I. Introduction. - Le développement des mesures spectrophotométriques dans l'ultra-violet lointain est étroitement lié à celui des sources; celles-ci sont de types divers suivant la région spectrale et la nature des mesures à effectuer [1]. Une des grosses difficultés rencontrées dans l'ultraviolet lointain est due au manque de matériaux transparents pour la réalisation de fenêtres. Le FLi clivé permet d'atteindre $1050 \AA$. En dessous on ne peut utiliser que des pellicules très minces de quelques centaines d'A épaisseur (collodion, $\mathrm{SiO}$, certains métaux [2]), mais ces pellicules absorbent encore une partie importante du rayonnement et ne peuvent pas supporter une pression supérieure à quelques torrs [3]; elles sont d'emploi peu pratique. On préfère généralement utiliser des sources sans fenêtre. Mais lorsqu'un gaz est nécessaire au fonctionnement de celles-ci, il se répand dans l'appareil dispersif (monochromateur par exemple) et dans les appareils de mesure; sa présence est d'autant plus gênante que sa pression est plus élevée. Il peut absorber certaines radiations ou gêner le fonctionnement des récepteurs (photomultiplicateur sans fenêtre par exemple). Le problème se pose particulièrement lorsqu'on désire faire des mesures dans une enceinte à ultravide $\left(10^{-10}\right.$ torr $)$ placée à la fente de sortie d'un monochromateur (sans fenêtre entre la source et cette enceinte). On peut limiter la diffusion du gaz en utilisant des pompages différentiels devant les fentes du monochromateur, mais on a toujours intérêt à utiliser des sources fonctionnant à une pression aussi faible que possible. Les décharges condensées dans les tubes capillaires [4] ou entre électrodes métalliques [5] initiées par une étincelle glissante peuvent fonctionner à basse pression; toutefois elles n'ont généralement pas une stabilité aussi bonne que les décharges dans un gaz alimentées par une tension continue bien stabilisée.

Dans le but d'effectuer des mesures aussi précises que possibles de constantes optiques de matériaux évaporés en ultravide $[6,7]$, nous avons été amenés à effectuer une étude des sources sans fenêtre de type Penning, dans lesquelles le champ magnétique permet d'entretenir la décharge même à très basse pression.

II. Principe des sources du type penning. - Le principe de la décharge de Penning [8] est simple ; il consiste à allonger les trajectoires des électrons et des ions dans un tube à décharge à l'aide d'un champ magnétique. La décharge peut alors se maintenir jusqu'à des pressions très basses (des jauges construites sur ce principe sont encore utilisables à des pressions inférieures à $10^{-12}$ torr). Pour une étude détaillée de ce phénomène voir par exemple [9]. Quelques essais pour utiliser la décharge de Penning comme source de lumière ont été effectués [10], mais la grosse difficulté réside dans l'érosion des cathodes et les dépôts qui peuvent en résulter; cette érosion peut être très rapide lorsque l'intensité dans la source est importante. (Un procédé d'évaporation sous vide est d'ailleurs basé sur ce principe [11].) Pratiquement la source doit comporter deux cathodes $C_{1}$ et $C_{2}$ portées au même potentiel et une anode A (Fig. 1) ; un champ d'induction $B$ est perpendiculaire aux cathodes et on établit une tension de quelques milliers de volts entre cathodes et anode. S'il règne une pression de $10^{-4}$ à $10^{-1}$ torr dans la source, il s'établit une colonne lumi- 
neuse $D$ qui peut être assez intense pour servir de source. Les spectres émis sont ceux d'atomes plusieurs fois ionisés et sont riches en raies.

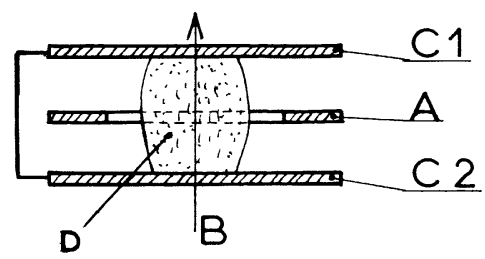

Fig. 1. - Principe de la décharge de Penning.

Plusieurs montages sont possibles; on peut observer la décharge dans le sens de l'induction $B$ ou perpendiculairement à cette induction. Il peut être commode de mettre les cathodes ou l'anode à la masse du monochromateur. Il est préférable de refroidir les éléments de la source par un courant d'eau. La géométrie doit être étudiée de manière à limiter la vaporisation du métal sur les isolants séparant l'anode des cathodes et vers la fente d'entrée du monochromateur. On constate aisément que les dépôts sont considérablement diminués sur les éléments qui ne sont pas en vision directe des cathodes à l'endroit de la décharge. La quantité de métal vaporisé dépend de la nature du gaz utilisé dans la source; avec les atomes lourds comme l'argon, l'érosion est plus rapide qu'avec les molécules légères comme l'hydrogène pour une intensité comparable. Elle dépend aussi de la nature des cathodes ; le tantale apparaît comme particulièrement favorable ([11], p. 415); nos essais ont montré que les cathodes recouvertes de tantale donnent en effet de bons résultats.

Ces sources peuvent être utilisées avec une forte intensité (500 mA ou plus) si l'on dispose d'un miroir torique placé entre la source et la fente d'entrée du monochromateur pour diminuer la pulvérisation vers le réseau; dans le cas contraire, on a intérêt à les utiliser à faible intensité. Il est alors nécessaire d'amplifier suffisamment le signal à la sortie du photomultiplicateur de mesure. Nous avons obtenu de bons résultats avec le photomultiplicateur sans fenêtre Bendix M 306 à très faible bruit de fond $\left(10^{-12}\right.$ ampère), suivi d'un électromètre Keithley 416 ou avec un photomultiplicateur EMI $6255 \mathrm{~S}$ utilisé avec du terphényl comme substance fluorescente et un amplificateur synchrone PAR type 121. Dans ce dernier cas, le choppage de la lumière peut être effectué mécaniquement à l'aide d'un disque tournant percé de trous placé dans le vide derrière une des fentes du monochromateur ; on peut aussi utiliser un choppage électronique de la source à l'aide d'une triode de puissance (par exemple EIMAC 304 T. H.) dont la grille est commandée par un générateur de signaux basse fréquence. Nous avons utilisé les deux méthodes. La seconde évite toute vibration et permet d'obtenir facilement toutes les fréquences désirées.
III. Quelques types de réalisation. - Plusieurs types ont été réalisés et étudiés, avec observation dans le sens de l'induction $B$ ou perpendiculairement à $B$, avec cathodes ou anode à la masse ; nous ne décrirons que les deux modèles actuellement utilisés couramment pour nos mesures $[6,7]$.

1) DÉCharge OBSERVÉE DANS LE SENS DE L'INDUCTION. - Cette source est schématisée figure 2. L'induction est produite par un puissant aimant en fer à cheval qui permet d'obtenir environ 600 gauss au centre de l'anode. Les pièces polaires en fer 2 jouent en même temps le rôle de cathodes; leur forme est

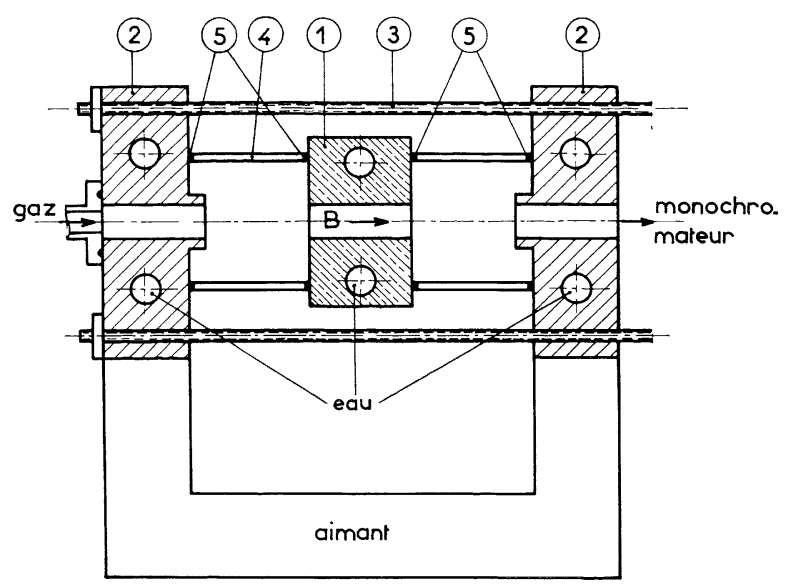

Fig. 2. - Source à décharge observée dans le sens axial.

étudiée pour favoriser la concentration du flux magnétique sur l'axe; elles sont à la masse et isolées de l'anode 1 en aluminium par deux manchons de quartz 4 qui reposent sur des joints d'indium 5 ; des tiges filetées extérieures 3 joignant les pièces 2 assurent la rigidité de l'ensemble. Le pompage est effectué à travers la fente du monochromateur.

Cette source est utilisée à faible intensité (5 à $10 \mathrm{~mA}$ ) car il est difficile d'éviter la pulvérisation sur l'isolant et vers la fente du monochromateur. Elle est toutefois relativement stable et brillante par suite de la concentration de la décharge lumineuse aux environs de l'axe. Il faut cependant nettoyer assez fréquemment les manchons de quartz 4 . De plus les fuites magnétiques peuvent être gênantes si l'on doit utiliser un récepteur aux environs de la source. Des essais sont actuellement en cours pour éliminer la pulvérisation et remplacer l'aimant par un solénoïde.

2) DÉCHARge OBSERVÉE TRANSVERSALEMENT AVEC CATHODES A LA MASSE (Fig. 3). - Le corps de la source est taillé dans un bloc d'aluminium 1. L'induction (600 gauss au centre de la décharge) est créée par deux barreaux cylindriques aimantés 2 collés à l'araldite dans le bloc d'aluminium 3. Les parties utiles des cathodes sont constituées par deux pastilles de fer doux 4 placées sur les extrémités internes des aimants et recouvertes d'aluminium ou de tantale 5 ; elles sont 
facilement interchangeables. L'anode 6 isolée par la pièce de téflon 8 est taillée dans une lame d'aluminium qui porte une ouverture rectangulaire 7 permettant l'observation de la décharge 11 et joue le rôle de diaphragme pour limiter la vaporisation vers la fente d'entrée du monochromateur. L'anode et les cathodes sont refroidies par un courant d'eau 9 ; des ouvertures latérales 12 permettent le pompage, une observation latérale de la décharge, l'introduction du gaz et la mesure de la pression. La fixation est assurée par les perçages 10 et l'étanchéité par un joint torique 13 .

Cette source est robuste, légère, stable et peu encombrante. Les fuites magnétiques sont très faibles aux environs de la source. Nous l'utilisons avec une intensité de 50 à $100 \mathrm{~mA}$. Il est possible de faire fonctionner cette source avec un double plasma en utilisant quatre aimants cylindriques au lieu de deux (Fig. 3) et en fermant le champ avec deux pièces de fer doux 14 .

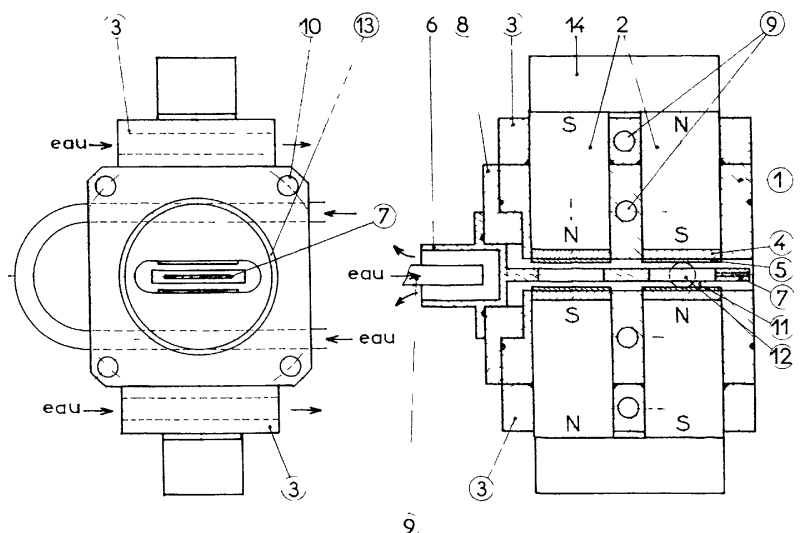

FIG. 3. - Source à décharge observée transversalement avec cathodes à la masse.

Cette disposition utilisée dans certains types de jauge (ALCATEL) diminue encore les fuites magnétiques, mais la stabilité est à peu près aussi bonne avec un seul plasma. C'est ce type de source qui nous semble présenter pour l'instant le plus d'avantages.

IV. Spectres obtenus. - Ces sources sont tout à fait comparables en ce qui concerne la pression, la stabilité et la nature des spectres obtenus. Pour obtenir une décharge brillante et stable, la pression est généralement comprise entre $10^{-3}$ et $10^{-2}$ torr.

Les figures 4 et 5 représentent les raies les plus intenses des spectres obtenus avec un monochromateur à réseau concave en incidence normale avec $\mathrm{H}_{2}, \mathrm{~N}_{2}$, $\mathrm{O}_{2}, \mathrm{He}, \mathrm{Ne}$ et $\mathrm{A}$; on a porté en ordonnées les courants à la sortie du photomultiplicateur suivant une échelle logarithmique; la valeur absolue de ces courants n'est qu'indicative ; elle dépend en effet de nombreux facteurs (photomultiplicateur, largeur des fentes, intensité de la source...). Nous avons tenté d'identifier les raies les plus intenses de ces spectres; les chiffres romains placés après les symboles des éléments indiquent si la radiation est attribuée à l'atome neutre ou

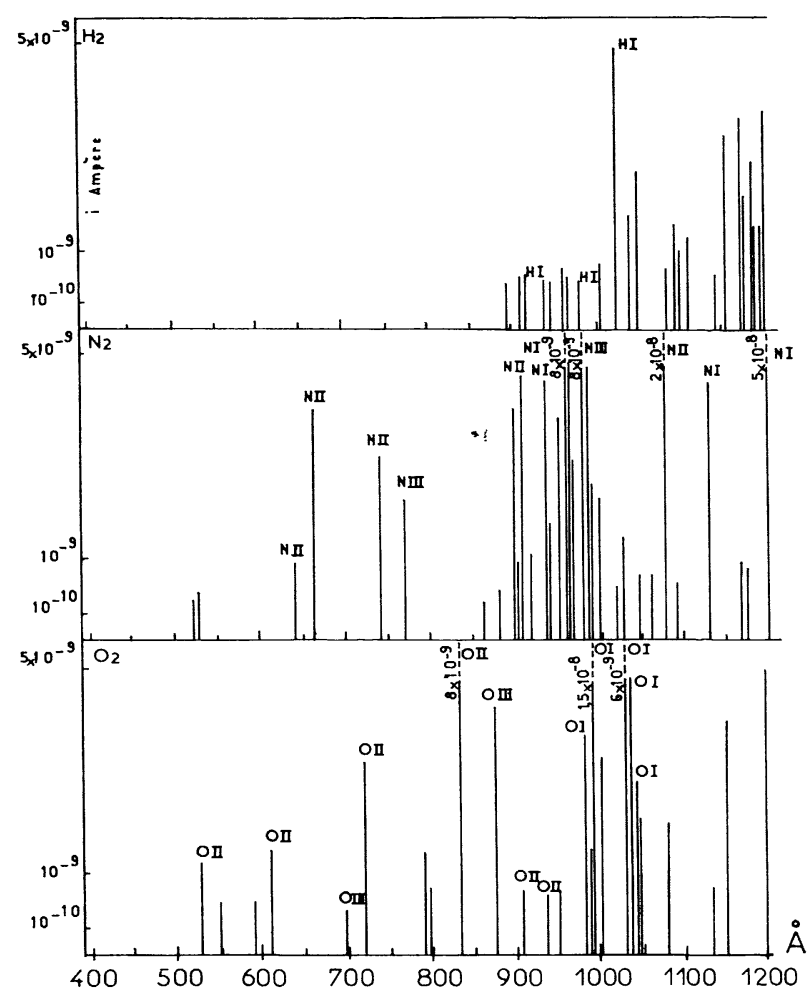

FIG. 4. - Spectres des décharges observées avec quelques gaz diatomiques (seules les principales raies ont été représentées).

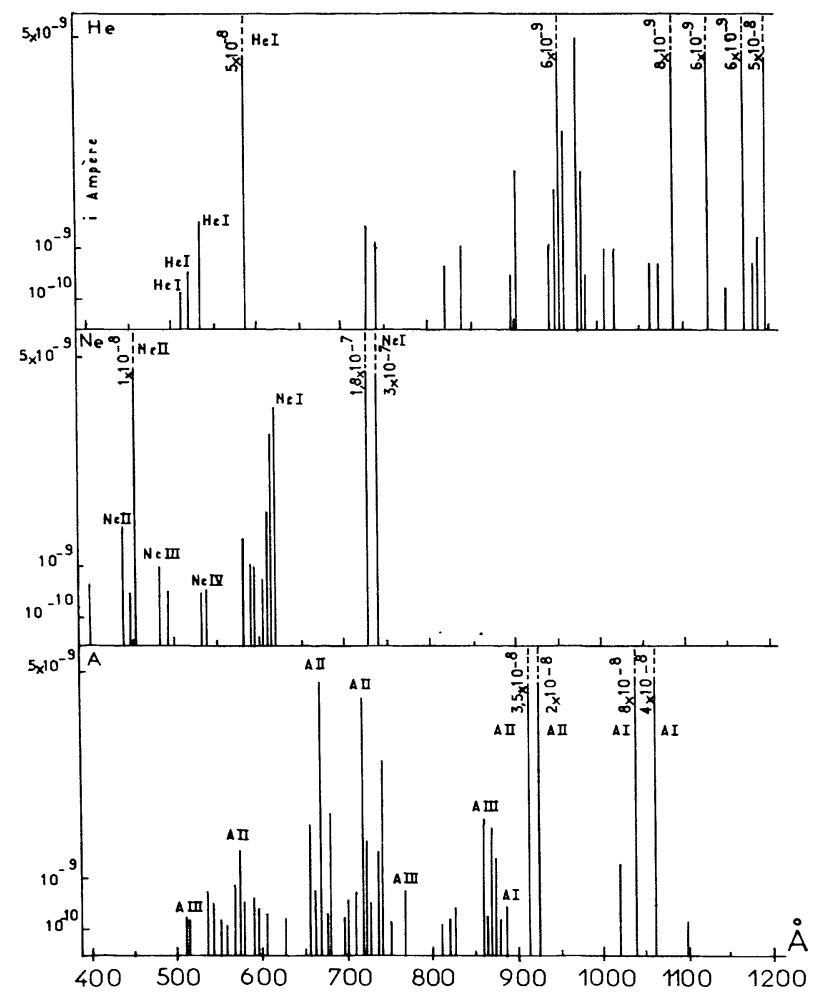

Fig. 5. - Spectres des décharges observées avec quelques gaz rares (seules les principales raies ont été représentées).

aux ions de différents degrés d'ionisation. Certaines raies n'ont pas été identifiées. Elles pourraient être 
dues à l'excitation des métaux (le fer par exemple) ou de produits de décomposition des isolants (le carbone par exemple). Au point de vue stabilité, lorsque la source a atteint son régime d'équilibre, les variations sur le sommet des raies ne dépassent pas $0,5 \%$ pendant 15 minutes ou $1 \%$ pendant 2 heures.

Conclusion. - Le développement de ce type de sources a probablement été gêné par les inconvénients dus à la vaporisation des cathodes mais lorsque la géométrie est bien étudiée et si les intensités utilisées ne sont pas trop fortes, cette vaporisation peut rester faible dans la direction d'observation. Ce type de source peut alors rendre de grands services aux expérimentateurs qui ont besoin d'une source stable fonctionnant à faible pression.

\section{Bibliographie}

[1] Robin (S.), Revue du GAMS, 1966, 3-4, 127.

[2] Hunter (W. R.), Angel (D. W.) et Tousey (R.), Appl. Optics, 1965, 4, 891.

[3] De Reilhac (L.), Damany-Atoin (N.) et Romand (J.), Spectr. Acta, 1969, 25 A, 19.

[4] Morlais (M.) et Robin (S.), Appl. Optics, 1967, 6, 409.

[5] Damany (M.), Roncin (Y.) et Damany-Astoin (N.), Appl. Optics, 1966, 5, 297.

[6] Daude (A.), Priol (M.) et Mme Robin (S.), $C . R$. Acad. Sci., Paris, 1966, 263, 1178.
[7] Daude (A.), Priol (M.) et Robin (S.), C. R. Acad. Sci., Paris, 1967, 264, 1489.

[8] Penning (E. M.), Physica, 1937, 4, 71.

[9] Schuurman (W.), Physica, 1967, 36, 136-160.

[10] Deslattes (R. D.), Peterson (T. J.) et Tomboulian (D. M.), J. O. S. A., 1963, 53, 302.

[11] Holland (L.), Vacuum deposition of thin films, Chapman \& Hall Ltd, Londres, 1961. 